\title{
Method of calculation of system reliability on the basis of construction of the logical function of the system
}

\author{
Dilshod Okhunov $^{1 *}$, Mamatjon Okhunov ${ }^{2}$, Mukaddas Akbarova ${ }^{2}$ \\ ${ }^{1}$ Fergana Branch of the Tashkent State University of Information Technologies named after Muhammad al-Xorazmiy. Fergana, \\ Uzbekistan \\ ${ }^{2}$ Fergana polytechnical institute. Fergana, Uzbekistan
}

\begin{abstract}
The article considers methods of calculating the reliability of systems using the logical functions of the system on the basis of the structural scheme of the system. And also the appliance of this method to calculate the probability of hacking the system based on the logical function of it.
\end{abstract}

\section{Introduction}

As it's known, the reliability of the system is the ability of it to perform a set of specified algorithms for processing information with a specified reliability or the property of the system to maintain its own operability at some point in time in the specified operating conditions.

Hacking system, unlawful penetration into it, in the vast majority of cases over time should lead from a lownoticeable to an impressive increase in the number of failures in the system, due to (even minor) fluctuations in the operating modes of the system [1].

This assertion is quite obvious if illegal penetration is carried out, for example, using special electromagnetic channels, which immediately leads to the passage beyond the permissible limits of such parameters as the current strength at the penetration node or voltage that are included in the number of necessarily controlled parameters of the system. It is well known that a high level of reliability (0.99999) requires absolutely accurate finding of any controlled parameter within a certain tolerance, which is controlled by the object management system $[2,3,4]$. Failure is a fundamental concept of reliability theory - a sudden or gradual (metrological) hacking into a system will necessarily react to this by increasing the failure rate $\lambda$, equal to the average number of failures per unit time. But the reliability of the system $\left(\mathrm{P}=e^{-\lambda t}\right)$, as can be seen, with increasing $\lambda$, will decrease.

\section{Methodology}

It is known, that calculating the reliability of a system has been used very successfully for a long time, based on the construction of a structural diagram of the system (block diagram) appropriate to the system itself $[5,6,7]$.
According to this, the so-called logical function of working capacity corresponding to this scheme is calculated, from which, after a number of mathematical operations (by using logical symbols $\wedge, \vee,-$ ), the reliability of the system is calculated.

The heart of our calculation methodology is the representation of our system by a block diagram, but as a parameter of each block, we will not consider the value of its reliability $\left(P_{\mathrm{b} / \mathrm{o}}\right)$, we will analyze the probability of hacking this block $\left(\mathrm{P}_{\text {break }}\right)$, which is naturally calculated in advance. The resulting logical "hacking" function is processed completely analogously to the logical function of working ability, widely used in reliability theory.

We will write down an algorithm of sequence of operations for calculating the reliability of a complex cybernetic system:

1. To formulate verbally the condition of the operability of the system, product, device.

2. Based on the verbal formulation, write down the logical function of working capacity.

3. Using a set of allowed postulates that correspond to the algebra of logic, try to convert, simplify the logical function $\mathrm{F}$, bring it to a form that can not be simplified, or, in other words, minimized.

4. In $F$ replace logical operations with their corresponding arithmetic, connected by signs $«+,-,{ }^{*} \ldots »$.

5. In the arithmetic function FA, replace the simple events, connected by arithmetic signs, with their probabilities.

6. Using the formula for FA, calculate the probability of working capacity interests us, by replacing the corresponding probabilities with their numerical estimates.

\footnotetext{
${ }^{*}$ Corresponding author: mamatdilshod@rambler.ru
} 
This table of postulates is used in the calculation of complex systems.

1. $a \vee b=b \vee a$

2. $a b=b a$

3. $a \vee(b \vee c)=(a \vee b) \vee c$

4. $a \vee(b \wedge c)=(a \vee b)(a \vee c)$

5. $a(b \vee c)=a b \vee a c$

6. $a \vee a=1 ; a \vee 0=a, a \wedge 0=0$

7. $a * 1=a, a \vee 1=1$ the absorption law

$8 a * \bar{a}=0$

9. $a \vee a \bar{b}=a \vee b$

10. $a \vee a=a$

11. $a * a=a$

12. $\overline{(a \vee b)}=\overline{a b}$

13. $\overline{(a b)}=\bar{a} \vee \bar{b}$

14. $a b \vee a \bar{b}=a$

15. $a(a \vee b)=a$

16. $F_{l}(a, b, c, \ldots k)=$

$a F_{l 1}(1, b, c, \ldots k) \vee \bar{a} F_{l 2}(0, b, c, \ldots k)$

Each postulate can be associated with a certain structural scheme (and vice versa), for example:

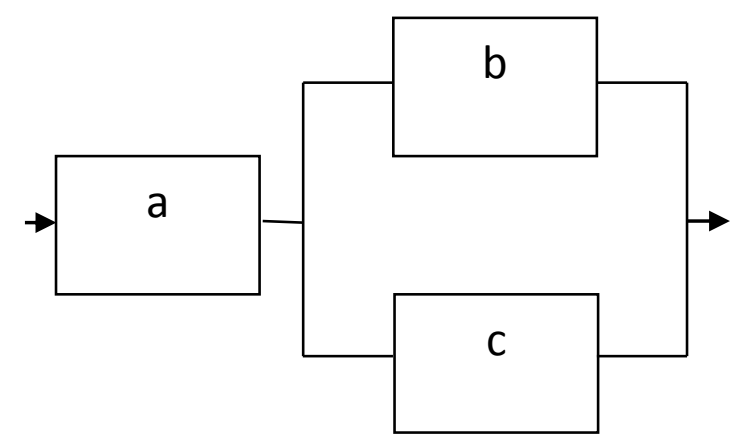

Fig. 1. Structural scheme example

In this case, postulate № $5 a(b \vee c)=a b \vee a c$ describes the condition of its operability.

It is clear that the system is operable, that means it transmits information, if $\mathrm{a}$ and $\mathrm{b}$, a and $\mathrm{c}$ or all three elements are operable, that's why this logical expression can be put into correspondence with this logical function:

$$
F_{\text {л }}=a b \vee a c \vee a b c=a b \vee a c=a(b \vee c)
$$

We have reduced logical function $F_{l}$ to an elementary, minimal form, from which we can make the transition to the arithmetic function $F_{A}$, using the standard set of arithmetic postulates:

$$
\begin{gathered}
a \vee b=a+b-a b \\
a \wedge b=a b \\
\bar{a}=1-a
\end{gathered}
$$

\section{Experimental results}

We need to define the probability of an operational state of the system if it is known that the reliability of the elements "a" and "b" are: $P_{a}=0,8$ and $P_{b}=0,8$.

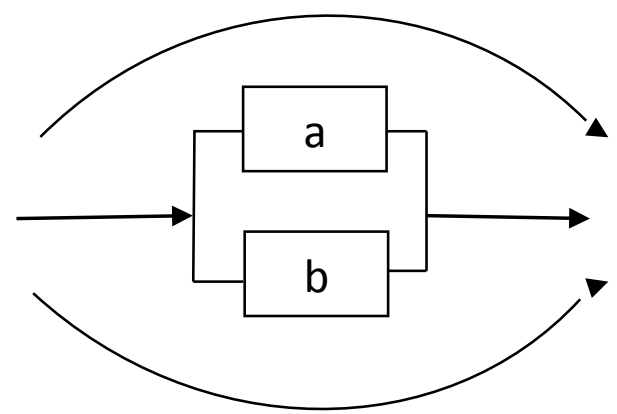

Fig. 2. The structural scheme at $P_{a}=0,8$ and $P_{b}=0,8$.

The first way to solve:

$$
\begin{gathered}
F_{l}=a \vee b \vee a b \\
P_{l}=a\{1 \vee b \vee 1 * \mathrm{~b}\} \vee \bar{a}\{0 \vee b \vee 0 * \mathrm{~b}\}=> \\
=>a * 1 \vee \bar{a} * b=>a \vee a b=>a \vee b
\end{gathered}
$$

Pass to the arithmetic logical function:

$F_{a}=a+b-a b=P_{a}+P_{b}-P_{a} * P_{b}=$

$=0,8+0,8-0,8 * 0,8=1,6-0,64=0,96$ 0.96

Reliability (probability of failure-free operation) is

The second way to solve:

$$
\begin{aligned}
F_{l}=a \vee b \vee a b & =>a(1 \vee b) \vee b(1 \vee a)=>a * 1 \vee b * 1 \\
& =>a \vee b
\end{aligned}
$$

Passing to the arithmetic logical function $F_{a}$, we obtain

$$
P_{b / o}=P_{a}+P_{b}-P_{a} * P_{b} \cong 0,96
$$

We calculate the reliability of the following scheme, if $P_{a}=P_{b}=P_{c}=0,8$.

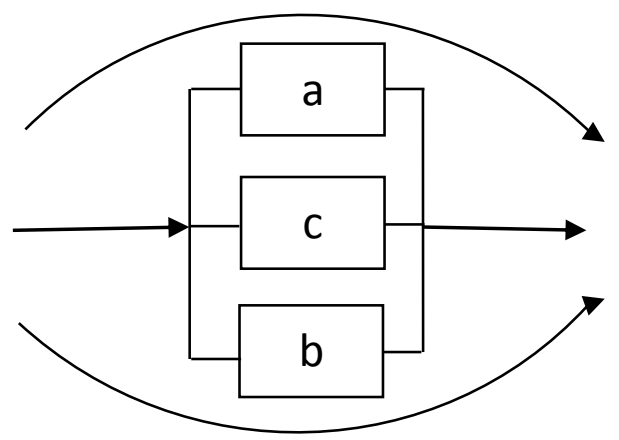

Fig. 3. The structural scheme at $P_{a}=P_{b}=P_{c}=0,8$

It is easy to see that

$F_{l}=a \vee b \vee c \vee a b \vee a c \vee b c \vee a b c$

Using the postulate 16 :

$F_{l}(\mathrm{a}, \mathrm{b}, \mathrm{c})=a F_{1}(1, \mathrm{~b}, \mathrm{c}) \vee \bar{a} F_{2}(0, \mathrm{~b}, \mathrm{c})$, we get:

$F_{l}(\mathrm{a})=a * 1 \vee \bar{a}(b \vee c)=>a \vee b \vee c$

We have obtained an elementary, primitive formula for a logical function. Turning to an arithmetic logic function

$$
F_{a}=a+b+c-a b-b c-a c+a b c
$$

substituting

$$
\begin{gathered}
a \rightarrow P_{a}=0,8, b \rightarrow P_{b}=0,8, c \rightarrow P_{c}=0,8, \text { arrive } \\
P_{b / o}=0,992 .
\end{gathered}
$$




\section{Conclusion}

Obviously, this principle of calculating reliability can be applied to the calculation of the probability of hacking of a certain system, if this system is represented as some structural scheme containing its channels of unauthorized access [8]. Consider the problem of calculating the probability of hacking the system based on the logical function of the system. This task is entirely in the class of tasks to model the threats of impact on all sorts of information sources: modeling of ways of penetration to the place of accumulation of information (for example, the head office); simulation of optical leakage channels (windows, doors, television embedded device); simulation of acoustic leakage channels; simulation of electronic leakage channels. This list can be continued further. However, the most diverse options are available for both the type of leakage channel (optical, electronic, etc.) and (in the general case of a random number) of simultaneously cracked channels [9]. If we try to clarify the probability of hacking the entire system, then this approach (from "private to general") is difficult to do, because the model of the entire system in these methods of hacking is absent from the outset, and the entire problem of estimating the probability of breaking (as well as the probability of hacking individual channels) to the class of weakly formable problems [10, $11]$.

However, if we look at the estimation of the probability of hacking the system, remaining within the framework of the system approach considered above, which can be implemented as some structural scheme of the system (where separate channels of illegal intrusion into the system act as separate blocks), then the problem of a general, the whole system can be solved with varying degrees of accuracy, depending on the correctness of the block diagram itself, composed of channels of hacking. This method consists in using a standard structural scheme of the system and compiling a logical function of operability, with its further standard processing. In this case, the reliability of each block of the structural diagram must be replaced with the probability of unauthorized penetration through this block $\left(P i_{\text {break }}\right)$. Further it is necessary to follow strictly on items (1-6) of the algorithm given above.

In conclusion, it should be noted that using this technique for calculating the logical function of the structural scheme, it is possible to calculate the reliability of more complex structural schemes - for example, schemes of a logical bridge, a triangle, etc.

\section{References}

1. Barsukov. V.S. Safety: technologies, means, services. Moscov, Kudishch-image Publ., (2001).

2. A.M. Polovko. The basis of the theory of reliability. - Moscov, "Nauka" Publ., (1964).

3. Ryabinin I.A. Reliability and safety of structurallydifficult systems. SPb.: publishing house of the St.Petersburg university, (2007).

4. Ryabinin I. A. Reliability of Engineering Systems. Principles and Analysis. - Moscov, Mir Publ., (1976).

5. Polovko A.M., Gurov S.V. Of the basis of the theory of reliability. - SPb.:BHV- Petersburg, (2006).

6. Polovko A.M., Gurov S.V. Of the Basis of the theory of reliability. A practical work. - $\mathrm{SPb}$.:BHVPetersburg, (2006).

7. Ryabinin I. A. Logiko-likelihood methods of research of reliability of structurally-difficult systems. Moscov, (1981).

8. Sovetov B.J. Yakovlev S.A. Modelling of systems. Moscov, Higher school Publ., 1989.

9. Titarenko G. A, Braga V.V., Vdovenko L.A. Information technologies of management. Moscov, UNITI Pabl., (2005).

10. Grinberg A.S., Gorbachev H.H, Teplykova A.A Protection of information resources of the government. Moscov, UNITI Pabl., (2003).

11. Grinberg A.S., Gorbachev H.H, Bondarenko A.S. Information of technology of management. Moscov, UNITI Pabl., (2004). 